\title{
LIBERTAD ECONÓMICA Y BIENESTAR INDIVIDUAL EN PERÚ
}

\author{
ECONOMIC FREEDOM AND INDIVIDUAL WELFARE IN PERU \\ VIADIM IR Rodríguez CAIro* \\ Docente Asociado de la Facultad de Ciencias Contables \\ Universidad Nacional Mayor de San Marcos - UNMSM / Lima-Perú \\ [Recepción: Agosto 2016/ Conformidad: Setiembre 2016]
}

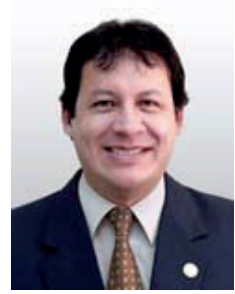

\section{RESUMEN}

La investigación permitió explicar por qué la libertad económica fue un factor que contribuyó a mejorar el bienestar individual en Perú durante el período 1970-2014. A partir de dicho objetivo, se realizó un análisis descriptivo sobre la relación entre las variables y se utilizaron dos aproximaciones basadas en el análisis inferencial: el coeficiente de correlación de Spearman y una estimación mediante Mínimos Cuadrados Ordinarios.

Siendo así, se obtuvieron como principales resultados que existe una relación entre el Índice de Libertad Económica y el Producto Bruto Interno per cápita, y que el grado de dicha relación es fuerte. Además, se constató que el sentido de la relación es positiva y estadísticamente significativa.

De este modo, se concluyó que la libertad económica es uno de los factores que influyó en el bienestar individual, y consiguientemente, en el bienestar general de la población. No obstante, la libertad económica es una condición necesaria pero no suficiente para determinar el bienestar. Es así que entre los principales aspectos que resaltan sobre la libertad económica en Perú, se encontraron: mejoras que se implementaron en la regulación del crédito, trabajo y negocios, comercio internacional y fortaleza financiera, así como avances más modestos en la estructura legal y el tamaño del gobierno.

\section{Palabras Clave:}

Libertad; económica; Influencia; Bienestar individual; Perú.

\section{ABSTRACT}

The research made possible to explain why economic freedom was a factor that contributed to improving individual well-being in Peru during the period 19702014. Based on this objective, a descriptive analysis was performed on the relationship between the variables, plus two approximations were used based on the inferential analysis: Spearman correlation coefficient and Ordinary Least Squares estimate.

Thus, the main results show that there is a relationship between the Index of Economic Freedom and the Gross Domestic Product per capita, and the strong degree of this relationship. In addition, it was found that the sense of the relationship is positive and statistically significant.

In this way, it was concluded that economic freedom is one of the factors that influenced individual welfare, and consequently, general well-being of the population. However, economic freedom is necessary but not a sufficient condition for determining well-being. Therefore, among the main aspects of economic freedom in Peru we found improvements in the regulation of credit, labor and business, international trade and financial strength, as well as more modest advances in the legal structure and size of government.

\section{Keywords:}

Economic freedom; Influence;Individual Welfare Peru.

\footnotetext{
*Economista y Abogado, Magíster en Economía, Magíster en Derecho y Doctor en Derecho y Ciencia Política. Email: vdriguezc@unmsm.edu.pe
} 


\section{INTRODUCCIÓN}

El presente estudio se enfoca en la relación entre la libertad económica y el bienestar de individual en Perú. Para tal efecto, se formula la siguiente interrogante: ¿Por qué la libertad económica es un factor que contribuye a mejorar el bienestar de individual en Perú en el período 1970-2014?

Desde tal perspectiva, la hipótesis que responde a la pregunta es: "A mayor libertad económica existe mayor bienestar individual en Perú en el período 1970-2014". Para tal efecto, se exponen los fundamentos teóricos de la libertad económica de Friedrich von Hayek, Milton Friedman, Ludwig von Mises y Amartya Sen. También se plantea la base teórica del bienestar de Stiglitz, Seny Fitoussi. Asimismo, se analizan los datos del Economic Freedom of the World 2016 Annual Report publicado por Fraser Institute de Canadá y del Producto Bruto Interno per cápita para Perú.

En lo esencial, tal como refiere la revisión efectuada por Sánchez, la mayoría de estudios analizados son de carácter longitudinal, los cuales permiten observar la relación entre libertad y crecimiento de diversos países, sin embargo, el presente estudio se orienta a conocer la relación particular que existe entre el bienestar de la población y el grado de libertad económica en nuestro país, separando de esta manera los efectos y distorsiones que puedan existir al incluir las características de otros países. Dentro de este contexto, la libertad económica es la facultad que tienen los individuos de elegir libremente la actividad económica que les permita obtener los medios adecuados para alcanzar sus respectivos fines. Las demás libertades sólo prevalecerán si se fortalece la libertad económica. En ese sentido, la libertad económica es un factor que contribuye a mejorar el bienestar de los individuos. Se fundamenta en el libre ejercicio de la actividad económica que mejor se adapte a las expectativas de cada persona.

Bajo tal premisa, el estudio recurre al enfoque cuantitativo, por ser probatorio a través de mediciones estadísticas. Por cierto, la principal limitación de la investigación es el número de observaciones disponibles, el cual restringe el uso de análisis estadísticos más avanzados. Dado que se cuenta con una serie anual de corta duración no se utilizan otras variables que podrían enriquecer el modelo para no perder grados de libertad.

Para una mejor comprensión del tema propuesto, el contenido se ha estructurado en cuatro partes. La primera comprende la fundamentación. En la segunda se plantea la metodología. La tercera abarcan los resultados. En la cuarta se aborda la discusión. Luego, se presentan las conclusiones. Finalmente, se da a conocer las referencias bibliográficas.

\section{FUNDAMENTACIÓN}

\section{Libertad económica}

La libertad económica, comprendida por un conjunto de elementos como: la posibilidad de elección, el intercambio voluntario, la libertad de constituir una empresa, la protección de la propiedad privada, entre otros, constituye un factor necesario para mejorar el bienestar individual y con ello, el de la sociedad.

La actividad económica proporciona los medios materiales necesarios para alcanzar todos nuestros objetivos (...). Si somos libres para elegir nuestros fines, es sólo porque también lo somos para elegir nuestros medios (...).La libertad económica ${ }^{1}$, por tanto, es una condición indispensable para todas las demás libertades, y la libertad de empresa es al mismo tiempo una condición necesaria y una consecuencia de la libertad personal (Hayek 2012: 321).

Dicho de otro modo, libertad económica es la facultad reconocida a los individuos de poder mejorar su bienestar a través del libre ejercicio de la actividad económica que mejor se adapte a sus fines, y a su vez, permitir que otros hagan lo mismo, de tal forma que cada persona participe en cualquier actividad económica de su elección ${ }^{2}$.Es así que para Hayek, la libertad económica es la más importante de todas las libertades,y por tanto, tiene mayor significado para la persona. Desde tal perspectiva, sin libertad

1. El significado de la libertad económica es que el individuo esté en posición de elegir la manera como desea integrarse en la totalidad de la sociedad. El individuo puede elegir su carrera: es libre de hacer lo que desea hacer (Mises 2008: 35).

2. El inciso 17 del artículo 2 de la Constitución Política del Perú establece el derecho fundamental de toda persona a participar, en forma individual o asociada, en la vida económica de la Nación. Así, toda persona natural o jurídica tiene derecho a decidir y elegir, con plena autonomía, la actividad económica que mejor se adapte a sus fines.

26/ QUipUiramavoc | Vol. 24(46) 2016 
económica no existe otro tipo de libertad. Por tal razón, resulta trascendente fortalecer la prerrogativa que tiene el individuo sobre el valor que genere a través del libre ejercicio de la actividad económica ${ }^{3}$.

En esa orientación, Milton y Rose Friedman (1980: 98-101) argumentan que una parte esencial de la libertad económica consiste en la facultad de escoger la manera en que vamos a utilizar nuestros ingresos: qué parte vamos a destinar para nuestros gastos y qué artículos vamos a comprar; qué cantidad vamos a ahorrar y en qué forma; qué monto vamos a regalar y a quién (...). Otra parte esencial de la libertad económica es la de utilizar los recursos que poseemos de acuerdo con nuestros propios valores: libertad para aceptar un empleo, para comprometerse en un negocio, para comprar y vender a cualquier persona (...). Añade que no somos libres de abrir un banco, entrar en la industria del taxi, o en la venta de electricidad o de servicio telefónico, o explotar una línea de ferrocarril, autobús o aérea, sin antes recibir una autorización de un funcionario gubernamental. No somos libres de participar en los mercados de capitales a menos que cumplamos muchas páginas de formularios que exige la SEC (Securities and Exchange Comission: Comisión de Valores y Bolsa). La libertad para ser dueño de propiedades constituye otra parte esencial de la libertad económica (...). Las restricciones a la libertad económica afectan.

Inevitablemente a la libertad en general, incluso en aspectos tales como la libertad de prensa y de expresión.

En ese entender, la idea primordial de Milton y Rose Friedman es que consideran a la libertad económica, como el derecho natural reconocido a los individuos para utilizar sus ingresos de la forma que consideren más conveniente.

Sustituir la economía de mercado por la planificación económica implica anular toda la libertad y deja al individuo un único derecho: el de obedecer (...). Tan pronto como se anula esa libertad económica que el mercado confiere a quienes en él participan, todas las libertades políticas, todos los derechos del hombre, se convierten en pura farsa (...).La gente es libre en aquella medida en que cada uno puede organizar su vida como considere mejor (Ludwig Von Mises 2011: 348-9).

Como resulta evidente, la libertad económica se pierde gradualmente con la mayor intervención del Estado a través del incremento o creación de nuevos tributos y excesivas regulaciones que terminan restringiendo las decisiones de los agentes económicos, obstaculizando de esta manera el emprendimiento de nuevas ideas de negocio, así como aquellas decisiones que permitan integrarse con la sociedad.

No obstante lo anterior, para Amartya Sen (2000: 25) la falta de libertad económica, en forma de extrema pobreza, puede hacer de una persona una víctima indefensa de la violación de otros tipos de libertad. Ejemplifica, afirmando que Kader $\mathrm{Mia}^{4}$, no habría necesitado ir a una zona hostil en busca de unos pequeños ingresos en aquella terrible época si su familia hubiera podido ser capaz de sobrevivir sin ellos. La falta de libertad económica puede alimentar la falta de libertad social, de la misma forma que la falta de libertad social o política también puede fomentar la falta de libertad económica.

En efecto, la extrema pobreza priva de capacidades básicas que implican mortalidad, desnutrición, analfabetismo, entre otros. De ahí que la libertad económica constituye una condición esencial para poder sobrevivir.

\section{2. Índice de Libertad Económica}

De acuerdo con el Reporte Anual delEconomic Freedom of the World(EFW) 2016 publicado porFraser Institute de Canadá,el Índice de Libertad Económica en el Mundo mide el grado en que las políticas y las instituciones de los países apoyan la libertad económica. Las piedras angulares de la libertad económica son la elección personal, el intercambio voluntario, la libertad para entrar en los mercados y competir y la seguridad de la persona y la propiedad privada propiedad. A continuación, se presenta una visión general de las cinco áreas principales ${ }^{5}$ :

\footnotetext{
3 Un análisis de los principios generales de la libertad se encuentra en: Rodríguez, V. (2015). Principios generales de una sociedad libre para fortalecer el orden espontáneo del mercado. Quipukamayoc. Página 125 y ss.

4 Hombre jornalero musulmán que tenía que ir a buscar trabajo para ganar algo porque su familia no tenía nada que comer. La consecuencia de su falta de libertad económica, fue la muerte que le sobrevino más tarde en el hospital.

5 El contenido de las cinco áreas se resume sobre la base del Reporte Anual del EFW 2016.
} 


\subsection{Tamaño del gobierno}

Los elementos de esta área indican el grado en que los países confían en el proceso político para asignar recursos y bienes y servicios. Cuando el gasto del gobierno aumenta en relación con el gasto de los individuos, las familias y las empresas, la toma de decisiones políticas se substituye por la elección personal y la libertad económica se reduce.

Mide el grado en que un país depende de la elección personal y los mercados en lugar de los presupuestos del gobierno y la toma de decisiones políticas. Por lo tanto, los países con bajos niveles de gasto público como porcentaje del total, un menor sector empresarial público y menores tasas de impuestos marginales obtienen las calificaciones más altas en esta zona.

\subsection{Sistema jurídico y garantía de los derechos de propiedad}

La protección de las personas y de sus bienes legítimamente adquiridos es un elemento central de la libertad económica y una sociedad civil. De hecho, es la función más importante del gobierno. Esta área se centra en este problema. Los ingredientes clave de un sistema jurídico coherente con la libertad económica son el estado de derecho, la seguridad de los derechos de propiedad, un poder judicial independiente e imparcial, y la aplicación imparcial y efectiva de la ley.

La seguridad de los derechos de propiedad, protegida por el estado de derecho, proporciona la base para la libertad económica y el funcionamiento eficiente de los mercados. La libertad de intercambio, no tiene sentido si los individuos no tienen derechos seguros a la propiedad, incluyendo los frutos de su trabajo. Cuando los individuos ylas empresas carecen de confianza en que se hará cumplir los contratos y los frutos de sus esfuerzos productivos, su incentivo para participar en la actividad productiva se erosiona. Quizás más que cualquier otra, esta área es fundamental para la asignación eficiente de los recursos. Los países con mayores deficiencias en esta área tendrán dificultades para prosperar independientemente de sus políticas en las otras cuatro áreas.

\subsection{Moneda sana}

El dinero "aceita" las ruedas del intercambio. La ausencia de una moneda sólida socava las ganancias del comercio. Tasas elevadas y volátiles de inflación distorsionan los precios relativos, alteran las condiciones fundamentales de contratos a largo plazo y hacen prácticamente imposible que los individuos y las empresas puedan planificar con sensatez para el futuro. Una moneda sana es esencial para proteger los derechos de propiedad y, por lo tanto, la libertad económica. La inflación erosiona el valor de los bienes mantenidos en instrumentos monetarios. Cuando los gobiernos financian sus gastos mediante la creación de dinero, están expropiando la propiedad y violando la libertad económica de sus ciudadanos.

Lo importante es que los individuos tengan acceso a una moneda sana: quien la ofrezca no hace mucha diferencia ${ }^{6}$. Si los banqueros pueden ofrecer ahorro y cuentas corrientes en otras monedas o si los ciudadanos pueden abrir cuentas bancarias en el extranjero, entonces el acceso a una moneda sana se incrementa y la libertad económica se expande.

Con el fin de obtener una puntuación elevada en esta zona, un país debe seguir las políticas y adoptar instituciones que llevan a la baja (y estables) las tasas de inflación y evitar las regulaciones que limitan la capacidad de utilizar monedas alternativas.

\subsection{Libertad de comercio internacional.}

En nuestro mundo moderno de alta tecnología y bajos costos de comunicación y transporte, la libertad de intercambio a través de las fronteras nacionales es un ingrediente clave de la libertad económica. Muchos bienes y servicios ahora se producen en el extranjero o contienen recursos del exterior. El intercambio voluntario es una actividad de suma positiva: ambos socios comerciales ganan y la búsqueda de la ganancia genera la motivación para el intercambio.

\footnotetext{
6 Pese a lo considerado en el Reporte Anual del EFW, Hayek(2014: 430) sostenía que aun cuando estaba convencido de que el moderno sistema de crédito bancario, en la forma como se ha desarrollado, requiere instituciones públicas tales como los bancos centrales, es discutible la necesidad o conveniencia de si dichas entidades (o los propios gobiernos) deben disfrutar del monopolio de emitir moneda (...)no veía que exista razón alguna para que el Estado prohíba en todos los casos el uso de otras clases de medios de cambio.
} 
Los aranceles y las cuotas son ejemplos evidentes de obstáculos que limitan el comercio internacional porque reducen la convertibilidad de las divisas. Controles sobre el tipo de cambio también obstaculizan el comercio internacional. El volumen de comercio también se reduce si el paso de las mercancías por aduanas es oneroso y consume mucho tiempo. A veces, estos retrasos son el resultado de la ineficiencia administrativa, mientras que en otros casos se da por la actuación de funcionarios corruptos que tratan de conseguir sobornos. En ambos casos, la libertad económica se reduce.

Con el fin de obtener una calificación alta en esta área, un país debe tener aranceles bajos, fácil remoción y una eficiente administración de aduanas, una moneda de libre convertibilidad y pocos controles a los movimientos de capital físico y humano.

\subsection{Regulación}

Cuando las regulaciones restringen la entrada a los mercados e interfieren con la libertad de participar en el intercambio voluntario, reducen la libertad económica. La quinta área del índice analiza las restricciones regulatorias que limitan la libertad de intercambio en los mercados de crédito, laborales y de productos. El primer componente refleja las condiciones del mercado de crédito interno. Los países que utilizan un sistema de banca privada para asignar el crédito a particulares y se abstienen de controlar las tasas de interés reciben las calificaciones más altas en este indicador.

Muchos tipos de regulaciones del mercado de trabajo vulneran la libertad económica de los empleados y los empleadores. Entre los más destacados están los salarios mínimos, las regulaciones de despido, la configuración centralizada de los salarios, la ampliación de los contratos de unión a terceros no intervinientes y el reclutamiento. Con el fin de obtener una alta calificación en el componente regulación del mercado de trabajo, un país debe permitir que las fuerzas del mercado determinen los salarios y establezcan las condiciones de contratación y despido y abstenerse de la utilización del servicio militar obligatorio.

Con el fin de obtener altas calificaciones en esta parte del índice, los países deben permitir que los mercados determinen los precios y abstenerse de actividades reguladoras que retarden la entrada de empresas y aumenten el costo de producción de los productos.

Tal como se aprecia en el siguiente Cuadro, se utilizan cuarenta y dos puntos (42) para la construcción de un índice global que mida el grado de libertad económica en cinco áreas generales. (Ver Cuadro $\mathrm{N}^{\circ} 01$ )

\section{Cuadro No 01}

Áreas, componentes y subcomponentes del Índice de Libertad Económica

\section{Tamaño del gobierno}
A. Consumo del gobierno
B. Transferencias y subsidios
C. Empresas e inversión pública

D. Tasa marginal máxima

(i) Tasa de impuesto sobre la renta marginal superior

(ii) Tasa de impuesto sobre el ingreso marginal superior y nóminas

II. Sistema jurídico y garantía de los derechos de propiedad
A. Independencia del poder judicial
B. Tribunales imparciales
C. Protección de los derechos de propiedad
D. Interferencia militar en el estado de dere- cho y la política
E. Integridad del sistema legal

\section{F. Aplicación de la ley de contratos}

G. Costos de regulación de la venta de bienes inmuebles

H. Confianza en la policía

I. Costos de la delincuencia

\section{Moneda sana}

\begin{tabular}{|ll|}
\hline A. Crecimiento del dinero & C. Inflación: año más reciente \\
B. Desviación estándar de la inflación & D. Libertad para abrir cuentas bancarias en monedas extranjeras \\
\hline
\end{tabular}




\section{Libertad de comercio internacional}
A. Aranceles
(i) Ingresos procedentes de los impuestos al comercio (\% del sector comercial)
(ii) La tasa arancelaria media
(iii) La desviación estándar de los tipos aran-
celarios
B. Reglamentos que obstaculizan el comercio
B. Reglamentos que obstaculizan el com
(i) Barreras comerciales no arancelarias
(ii) Costos de cumplimiento de la importa- ción y exportación

C. Tipos de cambio en el mercado negro

D. Controles de movimientos de capitales y de personas

(i) Propiedad extranjera / límites a la inversión

(ii) Control de capitales

(iii) Libertad de visitar otros países
A. Regulación del mercado de créditos
(i) Propiedad de los bancos
(ii) Crédito del sector privado
(iii) Control sobre las tasas de interés/ tasas de in- terés reales negativas
B. Regulación del mercado de trabajo
(i) Reglamentos de contratación y salario mínimo
(ii) Reglamentos de contratación y despido
(iii) Negociación colectiva centralizada
(iv) Reglamentos sobre las horas de trabajo
(v) Costo del despido de un trabajador

\section{Regulación}

Fuente: Gwartney, J. - Lawson R. - Hall J. (2016) Economic Freedom of the World 2016 Annual Report. Fraser Institute.

\section{Bienestar}

Como sostienen Stiglitz et al.(2013: 59) para precisar lo que significa bienestar hay que utilizar una definición con muchas dimensiones. A partir de la investigación académica y de un buen número de iniciativas concretas desarrolladas en todo el mundo, la comisión ${ }^{7}$ ha identificado las siguientes facetas clave que deben tenerse en cuenta simultáneamente:

a) nivel de vida material (ingresos, consumo y riqueza).

b) salud

c) educación

d) actividades personales (incluido el trabajo).

e) voz política (participar como ciudadano) y gobernanza (vigencia del Estado de Derecho)

f) conexiones y relaciones sociales (la gente con más contactos sociales evaluará mejor su vida, ya que muchas de las actividades más placenteras implican socialización).

g) entorno (condiciones presentes y futuras).

h) inseguridad física y económica.

Debe enfatizarse que los autores ${ }^{8}$ argumentan que la calidad de vida ${ }^{9}$ depende de la salud y la educación de las personas, sus actividades diarias (que incluyen el derecho a un trabajo y a una vivienda digna), su participación en los procesos políticos, el entorno social y natural en el que viven y los factores que dan forma a su seguridad personal y económica. Lo que realmente importa son las capacidades de las personas, es decir, las oportunidades a su alcance y su libertad de elección.

\footnotetext{
7 La comisión fue convocada por Nicolas Sarkozy, entoces presidente de la República Francesa, a principios del año 2008, en respuesta a la creciente preocupación acerca de la utilidad de las actuales mediciones del desarrollo económico -especialmente las basadas en las cifras del PBI-, y en respuesta también a la preocupación general sobre la relevancia de estas cifras como medida del bienestar de una sociedad, de la sostenibilidad económica, medioambiental y social.

8 Obra citada, página 60.

9 La calidad de vida es un concepto más amplio que la producción y el nivel de vida, pues incluye todos aquellos factores que influyen en lo que valoramos de la vida, más allá del aspecto material(Stiglitz et al. 2013: 113).
}

30/ QVIPURAMAYOC | Vol. 24(46) 2016 
Ahora bien, un indicador que ha sido utilizado por mucho tiempo para medir el desarrollo relativo de un países el Producto Bruto Interno (PBI) per cápita, el cual relaciona el PBI y la población de un país en un año determinado. Este indicador refleja la capacidad adquisitiva de los habitantes de un país. Pese a considerarse al PBI per cápita como un indicador del bienestar económico, presente algunos inconvenientes:

1) No reconoce las diferencias en la distribución del ingreso entre países.

2) Tiende a subestimar el nivel de vida de la población en aquellos países en los que la producción para el auto consumo es una parte importante del total producido.

3) No toma en cuenta la conservación del medio ambiente o el aprovechamiento sostenible de los recursos naturales.

\section{METODOLOGÍA}

\section{Alcance}

La investigación fue correlacional y explicativa. Correlacional porque tuvo como propósito evaluar la relación que existe entre la libertad económica y el Producto Bruto Interno per cápita para Perú. Explicativa debido a que se centró en explicar por qué se relacionan ambas variables.

\section{Diseño}

El estudio correspondió a un diseño no experimental pues no se ha construido alguna situación que intervenga a manera de tratamiento en los hechos estudiados; $\mathrm{y}$, de corte transversal pues se considera a las observaciones independientes del tiempo. A pesar de tratarse un corte transversal debido a serie temporal, pues los datos observados conciernen al seguimiento de las variables a lo largo del tiempo.

\section{Enfoque}

El enfoque fue cuantitativo por ser probatorio a través de mediciones estadísticas.

\section{Unidad de análisis}

Datos del Índice de libertad económica y el Producto Bruto Interno (PBI) per cápita de Perú para el período 1970-2014.

\section{Tratamiento de los datos y análisis de la información}

A partir de los datos obtenidos del Índice de Libertad Económica elaborado por el Instituto Frasery los datos del PBI per cápita del Banco Mundial para Perú, se aplicaron las siguientes técnicas estadísticas y métodos de estimación:

- Análisis descriptivo para determinar la relación entre el Índice de Libertad Económica y el PBI per cápita y la composición del Índice de Libertad Económica para Perú.

- Análisis inferencial a través de pruebas estadísticas para verificar la existencia y sentido de relación entre las dos variables de interés.

Dentro del análisis inferencial, cabe precisar que la principal limitante del estudio fue la disponibilidad de datos, lo cual restringió el uso de análisis estadísticos más avanzados. Dado que se contó con una serie anual de corta duración, tampoco se utilizaron variables que podrían haber enriquecido el modelo para no perder grados de libertad. Al mismo tiempo, si bien los datos corresponden a series de tiempo, dado que conciernen al seguimiento de las variables a lo largo del tiempo, se optó por tratarlos como un corte transversal, el cual opera sobre la base que las observaciones son independientes del tiempo debido a que las estimaciones como una serie de tiempo de corta duración llevaría a inferencias que afectan la interpretación de los resultados. Por tal motivo, a efectos de comprobar la hipótesis se recurrieron a dos aproximaciones: el coeficiente de correlación de Spearman y una estimación mediante Mínimos Cuadrados Ordinarios (MCO), utilizando el programa estadístico Stata.

10 El coeficiente de correlación de Spearman es una prueba estadística no paramétrica que sirve para verificar la relación entre dos variables. A diferencia del coeficiente de correlación de Pearson, esta prueba no requiere asumir normalidad en la distribución de las variables.

11 Cuando el número de observaciones es mayor a 20, se puede utilizar una aproximación a la distribución $\mathrm{t}$ - Student $\mathrm{T}=\mathrm{r} /\left(\left(1-\mathrm{r}^{2}\right) /(\mathrm{n}-2)\right)^{0.5}$. 


\section{Coeficiente de Correlación de Spearman}

Se verificó la existencia y sentido de relación entre las dos variables de interés mediante el coeficiente de correlación de Spearman ${ }^{10}$, calculado de la siguiente manera ${ }^{11}$ :

$$
r_{s}=1-\frac{6 \sum_{i=1}^{n}\left(d_{i}\right)_{i}^{2}}{n\left(n^{2}-1\right)}
$$

$d_{i}$ es la diferencia de rangos en el par $\mathrm{i}$

$\mathrm{n}$ es el número de pares

De manera que $r_{s} \in[-1,1]$ y -1 se refiere a una relación perfectamente negativa, 0 a que no existe relación y 1 a una relación perfectamente positiva. Además, el estadístico muestra el nivel de significancia de la relación. Así, si Prob $>|t|$ es menor al valor de significancia elegido $(0.01,0.05$ o 0.1$)$ se rechaza la hipótesis nula de independencia entre las variables, por lo que se concluye que existe una relación estadísticamente significativa entre las variables.

\section{Mínimos Cuadrados Ordinarios (MCO)}

Se estimó mediante un modelo MCO con errores estándar robustos la siguiente función:

$$
Y=F(X)
$$

En donde: $Y$ es el PBI per cápita en US\$

$\mathrm{X}$ es el Índice de Libertad Económica en el Mundo (escala de 0 a 10)

En la que se aplicó una transformación monótona creciente al término endógeno con el propósito de suavizarla serie. De esta manera la nueva función a estimar fue:

$$
\operatorname{Ln} Y=F(X)
$$

Así, el modelo a estimar fue el siguiente:

$$
\operatorname{Ln} Y=\alpha+\beta X+\mathcal{E}
$$

En donde: $\beta$ es el coeficiente asociado a $X$ $\varepsilon$ es el término de error aleatorio.

Por otro lado, la idoneidad del modelo fue evaluada por medio de los siguientes estadísticos en el Cuadro $\mathrm{N}^{\circ} 02$ :

\section{Cuadro No 02}

Descripción e interpretación de estadísticos

\begin{tabular}{|l|c|c|}
\hline Estadísticos & Descripción & Interpretación \\
\hline Prob $>\mathrm{F}^{12}$ & $\begin{array}{c}\text { Señala que las varia- } \\
\text { bles utilizadas me- } \\
\text { joran la bondad de } \\
\text { ajuste del modelo. }\end{array}$ & $\begin{array}{c}\text { El modelo es } \\
\text { mejor si tiende } \\
\text { a } 0\end{array}$ \\
\hline R2 & $\begin{array}{c}\text { Señala la bondad de } \\
\text { ajuste del modelo. }\end{array}$ & $\begin{array}{c}\text { El modelo es } \\
\text { mejor si tiende } \\
\text { a 1 }\end{array}$ \\
\hline
\end{tabular}

Fuente: Cameron, C. - Trivedi, P. (2009).

Elaboración propia.

De la misma manera, respecto de la variable exógena, obtener un $p$-value significativo asociado al $\beta$ identifica una relación estadísticamente significativa entre las variables $\mathrm{Xe}$ Y.Además el valor del $\beta$ señala el sentido de la relación entre ambas variables, por lo que $\beta>0$ muestra una relación positiva y $\beta<0$ lo contrario.

\section{RESULTADOS}

Hipótesis: "A mayor libertad económica existe mayor bienestar individual en Perú en el período 1970-2014".

De ahí que la Hipótesis fue evaluada, analizada e interpretada tomando en cuenta las siguientes variables:

- Variable endógena : bienestar individual

- Variable exógena : libertad económica

El Reporte Anual del Economic Freedom of The World (EFW) publicado por el Instituto Fraser ${ }^{13}$ es un instrumento valioso no sólo para medir la libertad económica de un país a través del tiempo, la cual toma en consideración diversos aspectos que contribuyen a explicar la libertad. También permite realizar investigaciones acerca de los efectos de la libertad económica sobre diversas variables de resultados, entre las cuales se encuentra el bienestar de quienes la experimentan o carecen de ella.

En la Tabla No 01, se observa la evolución de las variables endógena y exógena que se utilizaron para el análisis de las pruebas estadísticas.

12 Compara el modelo sin predictores (solo con el intercepto) con el modelo especificado.

13 El Instituto Fraser de Canadá publica los estimados sobre la base de información con un retraso de dos años.

32/ QUIPURAMAVOC | Vol. 24(46) 2016 
Tabla No 01

Perú: Índice de Libertad Económica y PBI per cápita 1970-2014

\begin{tabular}{|l|l|l|l|l|l|l|l|l|l|l|l|}
\hline & $\mathbf{1 9 7 0}$ & $\mathbf{1 9 7 5}$ & $\mathbf{1 9 8 0}$ & $\mathbf{1 9 8 5}$ & $\mathbf{1 9 9 0}$ & $\mathbf{1 9 9 5}$ & $\mathbf{2 0 0 0}$ & $\mathbf{2 0 0 1}$ & $\mathbf{2 0 0 2}$ & $\mathbf{2 0 0 3}$ & $\mathbf{2 0 0 4}$ \\
\hline Índice de Libertad Económica & 4,67 & 3,55 & 3,72 & 2,47 & 3,87 & 6,47 & 7,3 & 7,29 & 7,28 & 7,41 & 7,43 \\
\hline Área I: Tamaño del gobierno & 7,65 & 6,29 & 6,47 & 5,42 & 7,13 & 8,21 & 8,07 & 7,87 & 7,55 & 7,63 & 7,74 \\
\hline Área II: Sistema Jurídico & 1,36 & 1,15 & 3,77 & 2,23 & 2,93 & 4,76 & 3,94 & 4,23 & 4 & 4,64 & 4,65 \\
\hline Área III: Moneda sana & 5,94 & 4,87 & 1,62 & 0 & 1,25 & 5,13 & 8,79 & 9,49 & 9,72 & 9,56 & 9,61 \\
\hline Área IV: Libertad de comercio internacional & 3,76 & 1,89 & 3,29 & 1,3 & 4,07 & 7,9 & 8,35 & 8,37 & 8,13 & 8,2 & 7,96 \\
\hline Área V: Regulación & & & 3,46 & 3,39 & 3,96 & 6,34 & 7,37 & 6,51 & 7 & 7,02 & 7,16 \\
\hline PBI percápita (US\$) & $\mathbf{5 4 2}$ & $\mathbf{1 0 7 8}$ & $\mathbf{1 0 1 7}$ & $\mathbf{8 2 4}$ & $\mathbf{1 1 7 8}$ & $\mathbf{2 1 6 3}$ & $\mathbf{1 9 6 7}$ & $\mathbf{1 9 6 5}$ & $\mathbf{2 0 4 6}$ & $\mathbf{2 1 9 1}$ & $\mathbf{2 4 4 5}$ \\
\hline & $\mathbf{2 0 0 5}$ & $\mathbf{2 0 0 6}$ & $\mathbf{2 0 0 7}$ & $\mathbf{2 0 0 8}$ & $\mathbf{2 0 0 9}$ & $\mathbf{2 0 1 0}$ & $\mathbf{2 0 1 1}$ & $\mathbf{2 0 1 2}$ & $\mathbf{2 0 1 3}$ & $\mathbf{2 0 1 4}$ \\
\hline Índice de Libertad Económica & 7,39 & 7,42 & 7,4 & 7,52 & 7,52 & 7,6 & 7,59 & 7,53 & 7,34 & 7,35 \\
\hline Área I: Tamaño del gobierno & 7,71 & 7,73 & 7,79 & 7,85 & 7,51 & 7,53 & 7,56 & 7,59 & 7,46 & 7,4 \\
\hline Área II: Sistema Jurídico & 4,56 & 4,93 & 4,88 & 5,08 & 5,09 & 5,1 & 4,91 & 4,84 & 4,7 & 4,73 \\
\hline Área III: Moneda sana & 9,68 & 9,44 & 9,17 & 9,02 & 9,23 & 9,27 & 9,44 & 9,32 & 9,29 & 9,38 \\
\hline Área IV: Libertad de comercio internacional & 8,06 & 8,14 & 8,35 & 8,43 & 8,56 & 8,63 & 8,56 & 8,47 & 7,73 & 7,7 \\
\hline Área V: Regulación & 6,93 & 6,84 & 6,8 & 7,2 & 7,21 & 7,49 & 7,46 & 7,44 & 7,53 & 7,53 \\
\hline PBI percápita (US\$) & $\mathbf{2 7 1 4}$ & $\mathbf{3 1 4 4}$ & $\mathbf{3 6 1 1}$ & $\mathbf{4 2 4 5}$ & $\mathbf{4 1 7 9}$ & $\mathbf{5 0 5 6}$ & $\mathbf{5 7 3 2}$ & $\mathbf{6 3 9 0}$ & $\mathbf{6 6 1 0}$ & $\mathbf{6 5 4 9}$ & \\
\hline
\end{tabular}

Fuente: Fraser Institute, Banco Mundial Elaboración propia.

\section{Análisis descriptivo de la relación entreel Índice de Libertad Económica y el bienestar}

Como se observa la Figura N.o 01, entre los años 2000 y 2010 el Índice de Libertad Económica y el PBI Percápita per cápita siguieron un similar trayecto, denotando una estrecha relación entre los mismos. En cuanto a los últimos 4 años, si bien no se observó la misma tendencia entre los indicadores, se evidenció una ralentización en el crecimiento del producto per cápita, lo cual podría estar explicado por las expectativas de los agentes económicos, que pese a contar con un conjunto de normativas menos favorables, continuaron incrementando sus inversiones sobre la base de otros factores que consideran importantes aunque en menores proporciones que los años anteriores.

\section{Figura No 01}

Perú: Evolución del Índice de Libertad Económica y PBI per cápita 1970-2014

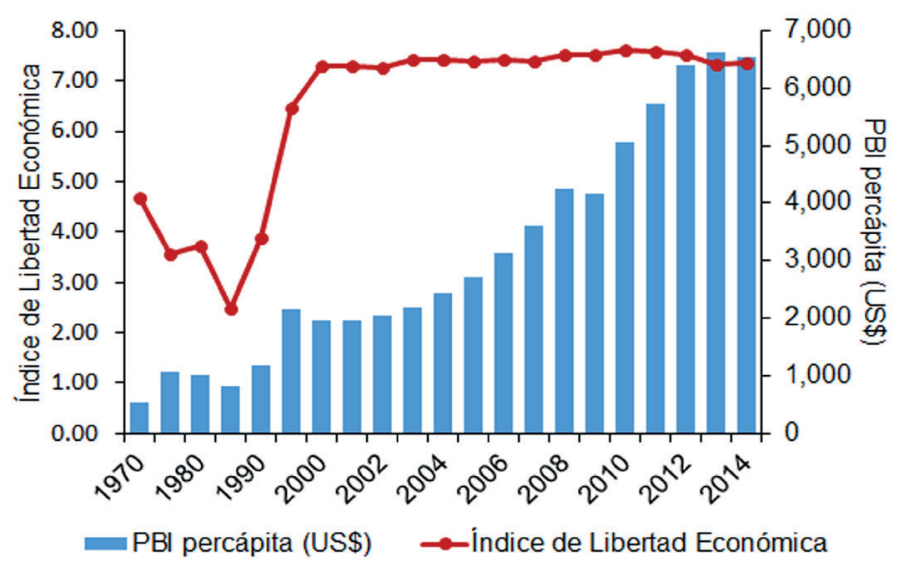

Fuente: Fraser Institute, Banco Mundial.

Elaboración propia. 


\section{Análisis de la estructura del Índice de Libertad Económica}

La Figura No 02 corresponde a los datos del grado de libertad económica de cada una de las cinco áreas generales (escala de 1 a 10): I) Tamaño del gobierno, II) Estructura legal, III) Moneda sana, IV) Libertad de comercio internacional, V) Regulación.

Un punto a destacar es la mejora considerable en los resultados entre el año de 1990 y los siguientes años, donde se implementaron medidas más liberales en aspectos de regulación, comercio internacional y fortaleza financiera, así como avances más modestos en la estructura legal y el tamaño del gobierno. Entre las principales medidas que explican esta mejora se pueden mencionar: la reformulación del régimen económico de la Constitución, la reforma del gasto público, la liberalización de los mercados, la firma de tratados de libre comercio, el fortalecimiento de los derechos de propiedad, el cumplimiento de contratos y la solidez de nuestra moneda.

\section{Figura No 02}

Perú: Estructura del Índice de libertad económica 1980-2014

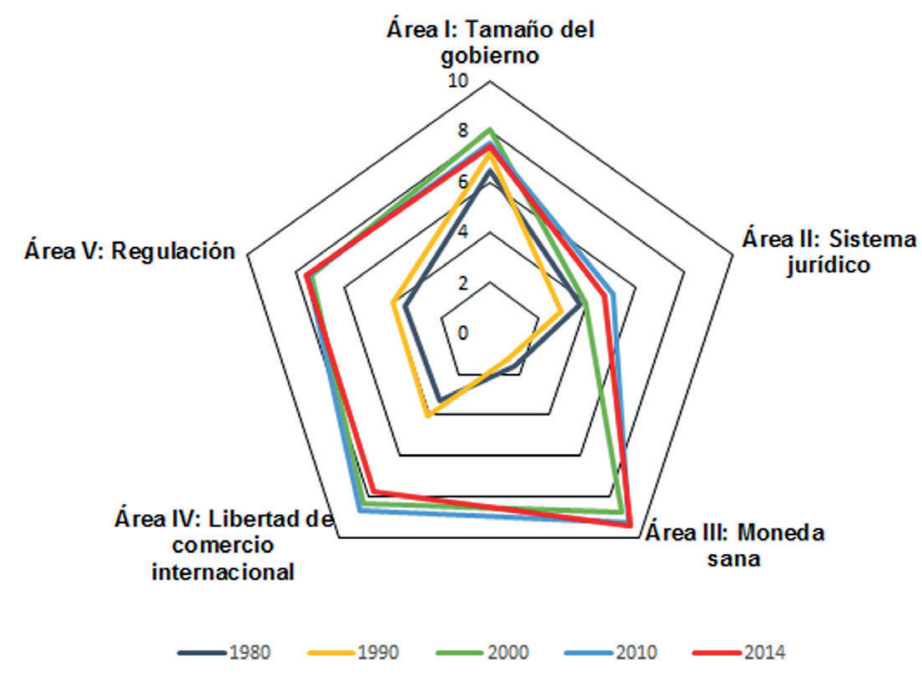

Fuente: Fraser Institute. Elaboración propia.

\section{Determinación de la correlación de Spearman}

En la Tabla No 02, se puede observar que a través del estadístico rs la relación entre las variables es positiva y fuerte, pues se acerca a 1 . Además, mediante Prob $>|t|$ se rechaza la hipótesis nula de independencia entre las variables, por lo que la relación estadística es significativa.

Tabla No 02

Estadísticos de Spearman

\begin{tabular}{|l|c|}
\hline \multicolumn{1}{|c|}{ Estadísticos } & Valor \\
\hline rs & 0.79 \\
\hline Prob $>|t|$ & 0.00 \\
\hline
\end{tabular}

Fuente: Elaboración propia.

\section{Estimación del modelo MCO}

La Tabla No 03, muestra los resultados de la estimación del modelo MCO. Por lo tanto, el modelo es bueno en términos de bondad de ajuste, pues el $\mathrm{R} 2$ tiende a 1 y a través de la prueba $\mathrm{F}($ Prob $>\mathrm{F})$ se muestra que la inclusión de la variable exógena sirve para mejorar el poder explicativo del modelo.

Tabla No 03

Estadísticos de Spearman

\begin{tabular}{|l|c|}
\hline \multicolumn{1}{|c|}{ Estadísticos } & Valor \\
\hline Prob $>\mathrm{F}^{14}$ & 0.00 \\
\hline $\mathrm{R} 2$ & 0.65 \\
\hline$\beta$ & 0.35 \\
\hline p-value $(\beta)$ & 0.00 \\
\hline
\end{tabular}

Fuente: Elaboración propia. 
Por último, $\beta$ nos señala que la relación entre las variables es positiva y estadísticamente significativa al $1 \%$ pues tiene un p-value igual a 0 .

\section{DISCUSIÓN}

La información del EconomicFreedom of The World (EFW) se utiliza en diversos artículos científicos de carácter empírico, en los cuales, los datos del Índice de Libertad Económicaconstituyen una variable exógena que explica el bienestar. Siendo así,los resultados de estas investigaciones muestran que aún en países de ingresos medios como el Perú, existe una relación entre la libertad económica y el bienestar (ingreso per cápita), a diferencia de lo hallado por Islam (1996). Sin embargo, no se encontraron estudios específicos sobre la libertad económica y bienestar para el caso peruano.

Del análisis realizado, de acuerdo con el EFW, Perú se sitúa en el puesto 52 entre 159 países en el 2014(frente al puesto 42 que ocupó el año 2013), detrás de países como Chile (13), Costa Rica (28), Guatemala (34), Panamá (37), El Salvador (42), Nicaragua $(45)^{15}$.

En estricto, los resultados (Tabla N..$^{\circ} 01$ y Figura N. $\left.{ }^{\circ} 01\right)$ muestran que si bien el Índice de Libertad Económica mejora ligeramente para nuestro país en el año 2014 (7,35) comparado con el 2013 (7,34), aún no se recupera de los niveles obtenidos en años anteriores. Así, el Perú se encuentra un poco estancado respecto de la medición del año 2010, donde alcanzó el nivel más alto $(7,6)$ del período 1970-2014.

Por otro lado, a diferencia de lo hallado en la revisión efectuada por Sánchez ${ }^{16}$, en donde muestra que la mayoría de estudios son de carácter longitudinal para observar la relación entre libertad y crecimiento de diversos países, el presente estudio se orienta a conocer la relación particular que existe entre el bienestar de la población y el grado de libertad económica en nuestro país, separando de esta manera los efectos y distorsiones que puedan existir al incluir las características de otros países.
De esta manera, aun con las limitaciones explicadas en la sección metodológica,el estudio a través de las pruebas estadísticas constata que la libertad económica y el bienestar en el Perú no son independientes y al mismo tiempo esta libertad económicatiene una participación positiva en la determinación del bienestar, debido a que añade valor explicativo al modelo que estima la relación. Lo importante del resultado obtenido, es que permite inferir la relevancia que tiene el libre ejercicio de la actividad económica a nivel individual sobre su bienestar, y consiguientemente, en el de la sociedad (Tablas N. ${ }^{\circ} 02$ y 03 ).

En este contexto, no se debe constreñir la libertad económica porque en función a ésta, los individuos y empresarios,a través del ensayo y error, van adaptando o corrigiendo sus decisiones al momento de participar en la actividad económica. Para ello, es vital contar con un gobierno limitado, que respete y garantice la libertad económica de los individuos y empresarios, ya que los incentivos de éstos tienden a desaparecer cuando el gobierno pretende fijar los fines al sector privado. Es más, cuando se obstaculiza el comercio, se crean y aumentan los impuestos en forma arbitraria o se modifican las leyes con bastante frecuencia se genera desconfianza para los negocios, hay menos productos disponibles en el mercado, $y$ sobre todo, se restringe la libertad económica de los agentes para participar en la actividad económica.

Sin perjuicio de lo señalado en los párrafos precedentes, la agenda de investigación en el futuro debería tratar de responder las siguientes interrogantes: ¿Cómo influye la interacción de otros factores en el bienestar individual?, ¿Cómo afectaron las medidas implementadas en el régimen económico del país sobre el grado de libertad económica, y éste a su vez, en el bienestar individual? ¿Cuál de las áreas del Índice de Libertad Económica explica de mejor manera el bienestar individual?, ¿Cómo se encuentra Perú comparado con los países latinoamericanos en cada una de las áreas del Î́ndice de Libertad Económica?

14 Compara el modelo sin predictores (solo con el intercepto) con el modelo especificado.

15 Hong Kong (9.03), Singapur (8.71), Nueva Zelanda (8.35), Suiza (8.25) y Canadá (7.98) son las naciones con mayor libertad económi ca del mundo, mientras que Argentina (4.81), Congo (4.80), Libya (4.58) y Venezuela (3.29) figuran en las últimas posiciones.

16 En: Libertad económica y crecimiento económico: teoría y evidencias. Fecha de consulta: 06/10/2016. Disponible en: <http://www. caminosdelalibertad.com/resources/uploads/pdf/20130528_140321_4concursoensayo_llugar.pdf>. 


\section{CONCLUSIONES}

1. Los datos del Índice de Libertad Económicapermitieron examinar el impacto de la libertad económica sobre el bienestar individual, medido a través del PBI per cápita. Se pudo constatar que durante el período 1970-2014, los peruanos mejoraron su bienestar a causa de la mayor libertad económica, tal como se presentó en la sección de Resultados. No obstante, se concluye que la libertad económica es una condición necesaria pero no suficiente para determinar el bienestar individual. En efecto, esta no es la única razón, ya que existen otras variables que explican el bienestar de las personas.

2. El estudio constata que la libertad económica y el bienestar en el Perú no son independientes y al mismo tiempo esta libertad económica tiene una participación positiva en la determinación del bienestar, debido a que añade valor explicativo al modelo que estima la relación. Lo importante del resultado obtenido, es que permite inferir la relevancia que tiene el libre ejercicio de la actividad económica a nivel individual sobre su bienestar, y consiguientemente, en el de la sociedad.

3. La investigación fija las bases para futuros estudios acerca de la influencia de otros factores en el bienestar individual, así como la implicancia de las medidas implementadas en el régimen económico del país sobre el grado de libertad económica, y finalmente, el análisis de las áreas del Índice de Libertad Económica para explicar de mejor manera el bienestar individual.

\section{REFERENCIAS BIBLIOGRAFICAS}

1. CAMERON, C. - TRIVEDI, P. (2009) MicroeconometricsusingStata. Texas. Stata Press Publication.

2. FRIEDMAN, M. - FRIEDMAN, R. (1980) Libertad de elegir. España. Ediciones Grijalbo S.A.

3. GWARTNEY, J. - LAWSON R. - HALL J. (2016)Economic Freedom of the World 2016 Annual Report.Fraser Institute.

4. HAYEK, F. (2014) Los fundamentos de la libertad. Novena edición (edición original 1959). España. Unión Editorial S.A.

5. HAYEK, F. (2012) Estudios de filosofía, política y economía. Segunda edición (edición original 1967). España. Unión Editorial S.A.

6. MISES, L. (2011) La acción humana. Décima edición (edición original 1949). España. Unión Editorial S.A.

7. MISES, L. (2008) Política económica. Segunda edición (edición original 1959). España. Unión Editorial S.A.

8. RODRÍGUEZ, V. (2015) "Principios generales de una sociedad libre para fortalecer el orden espontáneo del mercado”. En Revista: Quipukamayoc. pp 125 y ss.

9. SÁNCHEZ, I. "Libertad económica y crecimiento económico: teoría y evidencias". Disponible en: http://www.caminosdelalibertad.com/resources/uploads/pdf/20130528_140321_4concursoensayo_1lugar.pdf - Fecha de consulta: 06/10/2016.

10.SEN, A. (2000) Desarrollo y libertad. España. Editorial Planeta S.A.

11.STIGLITZ, J. - SEN, A. - FITOUSSI, J.(2013) Medir nuestras vidas. Las limitaciones del PIB como indicador de progreso. España. RBA Libros S.A. 